The Irrelevant Information Principle for Collective Probabilistic Reasoning

Adamcik, Martin and Wilmers, George

2013

MIMS EPrint: 2013.42

Manchester Institute for Mathematical Sciences

School of Mathematics

The University of Manchester

\footnotetext{
Reports available from: http://eprints.maths.manchester.ac.uk/

And by contacting: The MIMS Secretary

School of Mathematics

The University of Manchester

Manchester, M13 9PL, UK
} 


\title{
THE IRRELEVANT INFORMATION PRINCIPLE FOR COLLECTIVE PROBABILISTIC REASONING
}

\author{
Martin AdamČÍK And GeOrge Wilmers
}

Within the framework of discrete probabilistic uncertain reasoning a large literature exists justifying the maximum entropy inference process, $\mathbf{M E}$, as being optimal in the context of a single agent whose subjective probabilistic knowledge base is consistent. In particular Paris and Vencovská completely characterised the ME inference process by means of an attractive set of axioms which an inference process should satisfy.

More recently the second author extended the Paris-Vencovská axiomatic approach to inference processes in the context of several agents whose subjective probabilistic knowledge bases, while individually consistent, may be collectively inconsistent. In particular he defined a natural multi-agent extension of the inference process ME called the social entropy process, SEP. However, while SEP has been shown to possess many attractive properties, those which are known are almost certainly insufficient to uniquely characterise it. It is therefore of particular interest to study those Paris-Vencovská principles valid for ME whose immediate generalisations to the multi-agent case are not satisfied by SEP. One of these principles is the Irrelevant Information Principle, a powerful and appealing principle which very few inference processes satisfy even in the single agent context. In this paper we will investigate whether SEP can satisfy an interesting modified generalisation of this principle.

Keywords: uncertain reasoning, discrete probability function, social inference process, maximum entropy, Kullback-Leibler, irrelevant information principle

Classification: 03B42, 03B48, 68T37

\section{MOTIVATION}

In this paper we consider the following fundamental problem of discrete multi-agent probabilistic uncertain reasoning. We are interested in finding a general procedure which, given a finite set of agents, each possessing a subjective probabilistic knowledge base over a finite space of possible events, yields a single probability function or social probability function defined over that space of events, which optimally represents the joint knowledge of all the agents, and such that that general procedure satisfies some natural criteria derived from logical or rational considerations.

There are several initial assumptions we want to make. Firstly we assume that the probabilistic beliefs or "knowledge" of each particular expert is consistent with the laws of probability. Secondly all agents are assumed to have equal status, and the final social 
probability function should not depend on the order in which the agents' knowledge bases are considered.

We illustrate the motivation behind this idea by a toy two-agent example.

Imagine that two safety experts are dealing with a fault in a chemical factory producing nitrogen fertilizers. There is a problem with ammonia supply. Ammonia is stored in a tank connected to the rest of the factory by a valve which is operated by an electric circuit.

The first expert believes that there is a $40 \%$ chance of a mechanical fault on the valve. The second expert comes up with a different opinion that there is a $80 \%$ chance that there is a mechanical problem on that valve. Moreover, the first safety expert thinks that there is a $70 \%$ chance that there is a malfunction of the electric circuit. We suppose that both experts have no other knowledge related to this problem.

The joint beliefs (knowledge) of the two experts are inconsistent in this case. In practice, knowledge is usually incomplete and offers a lot of uncertainty; the first expert in above example has no knowledge about, for instance, the conditional probability that there is a fault on the the valve given that there is a fault on the electric circuit. The situation becomes more complicated once the second agent is considered whose knowledge is inconsistent with the knowledge of the first agent. Altogether we can ask the following question:

Question. How should a rational adjudicator whose only knowledge consists of what is related to him by the two experts above, evaluate the probability that both the valve and the electric circuit are faulty, based only on the experts' subjective knowledge specified above and without any other assumptions?

Assuming, as we do in this paper, that each agent's uncertain knowledge can be represented within the framework of probability theory, we can describe the knowledge of each expert by a set of possible probability distributions over four possible mutually exclusive cases: (1) a fault on the valve and a fault on the electric circuit, (2) a fault on the valve and no fault on the electric circuit, (3) no fault on valve and a fault on the electric circuit and (4) no faults on the valve or on the electric circuit (i.e. in this case there is a problem with something else). We can denote the corresponding probabilities that $(1),(2),(3)$ and (4) is true by real numbers $w_{1}, w_{2}, w_{3}$ and $w_{4}$ from the interval $[0,1]$ which sum to 1 . Based on the knowledge of the first expert $w_{1}+w_{2}=0.4$ and $w_{1}+w_{3}=0.7$. Any probability function $(x, 0.4-x, 0.7-x, x-0.1)$, where $x \in[0.1,0.4]$, is consistent with the knowledge of the first expert. Similarly, the second expert admits any $(x, 0.8-x, y, 0.2-y)$ where $x \in[0,0.8]$ and $y \in[0,0.2]$. This representation of the knowledge of the experts naturally abstracts from the complex nature of the actual problem. However we are not interested here in the particular manner in which this abstraction from the infinite complexity of a real world problem has been accomplished. Instead we will focus on the following narrower, abstract, but more clearly defined question: 
Question. Given two (or more) sets of probability functions corresponding to the knowledge bases of corresponding experts as in the above example, which single probability function best represents the combined probabilistic knowledge of the experts?

Naturally, we would like to find a general procedure doing this for any knowledge bases which satisfies some natural principles. We will formalize this idea in a general setting in the next section.

We should emphasize here that our reduction of the experts' knowledge to a theoretical question as above assumes that all the knowledge of an individual expert is incorporated in the formal represention of her knowledge base. This last assumption is sometimes referred to as the Watts assumption (see [10). In order to avoid confusion, it is clearly essential that this assumption be adhered to in any discussion of the general theoretical characteristics of a mode of probabilistic inference. Of course when applied to the formalisation of any real life problem considered by a human agent, the Watts assumption is always false in practice. This banal fact of life has historically bedevilled theoretical discussion of probabilistic inference, because it is often extremely hard to give illustrative real world examples of abstract principles of probabilistic inference without a philosophical opponent being tempted to challenge one's reasoning using implicit background information concerning the example which is not included in its formal representation as a knowledge base. This tendency to overlook the Watts assumption provides an inexhaustible supply of invalid arguments: see e.g. Jaynes [5] for an analysis of this phenomenon in relation to certain criticisms of the maximum entropy inference process.

\section{FORMALIZATION}

Let $L=\left\{a_{1} \ldots a_{h}\right\}$ be a finite propositional language where $a_{1}, \ldots, a_{h}$ are propositional variables. In our example $n=2, a_{1}$ stands for sentence "a fault on the valve" and $a_{2}$ stands for sentence "a fault on the electric circuit". By the disjunctive normal form theorem any $L$-sentence can by expressed as a disjunction of atomic sentences (atoms) and we will denote a maximal set of logically inequivalent atoms $\left\{\alpha_{1}, \ldots, \alpha_{J}\right\}$, where $J=2^{h}$, by $\operatorname{At}(L)$. The atoms of $\operatorname{At}(L)$ are thus mutually exclusive and exhaustive.

A probability function $\mathbf{w}$ over $L$ is defined by a function $\mathbf{w}: \operatorname{At}(L) \rightarrow[0,1]$ such that $\sum_{j=1}^{J} \mathbf{w}\left(\alpha_{j}\right)=1$.

A value of $\mathbf{w}$ on any $L$-sentence $\varphi$ may now be defined in the obvious way by setting

$$
\mathbf{w}(\varphi)=\sum_{\alpha_{j}=\varphi} \mathbf{w}\left(\alpha_{j}\right) .
$$

We will denote the set of all probability functions over $L$ by $\mathbb{D}^{L}$. For the sake of brevity we will often write $w_{j}$ instead of $\mathbf{w}\left(\alpha_{j}\right)$, but note that this notation makes sense only for atomic sentences $\alpha_{j}$. Given a probability function $\mathbf{w} \in \mathbb{D}^{L}$, a conditional probability is defined by Bayes's formula

$$
\mathbf{w}(\varphi \mid \psi)=\frac{\mathbf{w}(\varphi \wedge \psi)}{\mathbf{w}(\psi)}
$$


for any $L$-sentence $\varphi$ and any $L$-sentence $\psi$ such that $\mathbf{w}(\psi) \neq 0$ and is left undefined otherwise.

Now consider two distinct propositional languages $L_{1}=\left\{a_{1}, \ldots, a_{h_{1}}\right\}$ and $L_{2}=$ $\left\{b_{1}, \ldots, b_{h_{2}}\right\}$. Let $\operatorname{At}\left(L_{1}\right)=\left\{\alpha_{1}, \ldots, \alpha_{J}\right\}$ and $\operatorname{At}\left(L_{2}\right)=\left\{\beta_{1}, \ldots, \beta_{I}\right\}$. Then every atom of the joint language $L_{1} \cup L_{2}$ can be written uniquely (up to logical equivalence) as $\alpha_{j} \wedge \beta_{i}$ for precisely one $1 \leq j \leq J$ and precisely one $1 \leq i \leq I$. With only a slight abuse of notation, for an $L_{1} \cup L_{2}$-probability function $\mathbf{r}$ we will often write $r_{j i}$ instead of $\mathbf{r}\left(\alpha_{j} \wedge \beta_{i}\right)$, in a similar way as for an $L_{1}$-probability function $\mathbf{v}$ we write $v_{j}$ instead of $\mathbf{v}\left(\alpha_{j}\right)$.

Now notice that $\models \alpha_{j} \leftrightarrow \bigvee_{i=1}^{I} \alpha_{j} \wedge \beta_{i}$. Therefore, the marginal probability function whose $j$-th value is given by $\sum_{i=1}^{I} r_{j i}$ is the projection of an $L_{1} \cup L_{2}$-probability function $\mathbf{r}$ to the language $L_{1}$. We will denote it by $\left.\mathbf{r}\right|_{L_{1}}$. Similarly if $\Delta$ is a set of $L_{1} \cup L_{2}$-probability functions, we denote the set $\left\{\left.\mathbf{v}\right|_{L_{1}}: \mathbf{v} \in \Delta\right\}$ by $\left.\Delta\right|_{L_{1}}$. Also if $\mathbf{v}$ is an $L_{1}$-probability function and $\mathbf{w}$ is an $L_{2}$-probability function then the product function $\mathbf{v} \cdot \mathbf{w}$ defined by $\mathbf{v} \cdot \mathbf{w}\left(\alpha_{j} \wedge \beta_{i}\right)=v_{j} w_{i}$ is an $L_{1} \cup L_{2}$-probability function such that $\left.(\mathbf{v} \cdot \mathbf{w})\right|_{L_{1}}=\mathbf{v}$.

A (probabilistic) knowledge base $\mathbf{K}$ over $L$ is a set of constraints on probability functions over $L$ such that the set of all probability functions satisfying the constraints in $\mathbf{K}$ forms a nonempty closed convex subset $V_{\mathbf{K}}$ of $\mathbb{D}^{L} . V_{\mathbf{K}}$ may be thought of as the set of possible probability functions of a particular agent which are consistent with her subjective probabilistic knowledge base $\mathbf{K}$. In the sequel we shall loosely identify $\mathbf{K}$ with $V_{\mathbf{K}}$, and may also refer to such a $V_{\mathbf{K}}$ as a knowledge base. Note that the non-emptiness of $V_{\mathbf{K}}$ corresponds to the assumption that $\mathbf{K}$ is consistent, while if $\mathbf{K}$ and $\mathbf{F}$ are knowledge bases then the knowledge base $\mathbf{K} \cup \mathbf{F}$ corresponds to $V_{\mathbf{K} \cup \mathbf{F}}=V_{\mathbf{K}} \cap V_{\mathbf{F}}$. The set of all knowledge bases $V_{\mathbf{K}}$ over $L$ is denoted by $C L$.

In the toy example, the knowledge of the first expert can be represented by the knowledge base $\mathbf{K}$ which consist of a set of linear constraints on a probability function $\mathbf{w}=\left(w_{1}, w_{2}, w_{3}, w_{4}\right)$ defined over the atomic sentences $a_{1} \wedge a_{2}, a_{1} \wedge \neg a_{2}, \neg a_{1} \wedge a_{2}$ and $\neg a_{1} \wedge \neg a_{2}$. Then $\mathbf{K}=\left\{w_{1}+w_{2}=0.4, w_{1}+w_{3}=0.7\right\}$ and $V_{\mathbf{K}}=\{(x, 0.4-x, 0.7-$ $x, x-0.1): x \in[0.1,0.4]\}$.

Given $\mathbf{K} \in C L_{1}$ note that the underlying language $L_{1}$ is implicitly understood in the notation $V_{\mathbf{K}}$ which should more properly be denoted $V_{\mathbf{K}}^{L_{1}}$. Thus if $L_{1} \subset L$ then $\mathbf{K}$ is also in $C L$ and $V_{\mathbf{K}}^{L}=\left\{\mathbf{w} \in \mathbb{D}^{L}:\left.\mathbf{w}\right|_{L_{1}} \in V_{\mathbf{K}}^{L_{1}}\right\}$. For simplicity we shall normally just write $V_{\mathbf{K}}$ when the appropriate language is understood.

We now define the central notion which maps any given sequence of knowledge bases to a single probability function termed the social probability function for that sequence. A social inference process $\mathcal{S}$ defines for each $L$ and $n \geq 1$ a function

$$
\mathcal{S}_{L}: \underbrace{C L \times \ldots \times C L}_{n} \rightarrow \mathbb{D}^{L} .
$$

The number $n$ here intuitively represents the number of distinct agents or distinct sources of information.

The restricted notion $\mathcal{S}\left(\right.$ or $\mathcal{S}_{L}$ ) in the case of a single knowledge base or agent, i.e. when $n=1$, is simply called an inference process and the properties of such inference processes have been extensively studied by Paris, Vencovská and others (see [10], 12, [4], 11, [14, 5] ). 
As was noted above, a consistent knowledge base $\mathbf{K}$ yields a set $V_{\mathbf{K}}$ of possible probability functions consistent with $\mathbf{K}$. In the case of single agent with knowledge base $\mathbf{K}$ there are several possible procedures to choose a specific probability function from $V_{\mathbf{K}}$. However by the traditional possible worlds modelling or information theoretic arguments whose origins go back to nineteenth century statistical mechanics as in [1] or [5], the maximum entropy inference process $\mathbf{M E}$ has been justified as being optimal, where $\mathbf{M E}_{L}(\mathbf{K})$ is defined as that unique probability function $\mathbf{w}$ in $V_{\mathbf{K}}$ which maximizes the Shannon entropy $H(\mathbf{w})$ of $\mathbf{w}$ given by

$$
H(\mathbf{w})=-\sum_{j=1}^{J} w_{j} \log w_{j} .
$$

$H$ is a strictly concave function and therefore it attains a unique maximum over any nonempty closed convex region $V_{\mathbf{K}}$ of $\mathbb{D}^{L}$.

A quite different justification for $\mathbf{M E}$ to the traditional ones was described in [14] by Johnson and Shore. Their work was developed by Paris and Vencovská in [12] where they showed that a list of principles based on symmetry and consistency uniquely characterises ME. It therefore seems fruitful to look at the axiomatic approach also in the more general context of a social inference process. Accordingly we may ask:

What general principles should a social inference process $\mathcal{S}$ satisfy in order to ensure that for any given knowledge bases, and in the absence of any other information, $\mathcal{S}$ chooses a social probability function according to rational criteria?

We might hope that ultimately such a set of rational principles may determine uniquely a particular social inference process $\mathcal{S}$.

\section{LANGUAGE INVARIANCE AND IRRELEVANT INFORMATION}

In this section we examine how certain fundamental invariance principles formulated by Paris and Vencovská for an inference process (see [10]) can be extended to the notion of a social inference process.

An obvious question we need to ask regarding social inference processes is whether they depend on the choice of a particular propositional language $L=\left\{a_{1}, \ldots, a_{h}\right\}$. For fixed $\mathcal{S}, L$, with $\varphi \in S L$ and $\mathbf{K}_{1}, \ldots, \mathbf{K}_{n} \in C L$ consider $\mathcal{S}_{L}\left(\mathbf{K}_{1}, \ldots, \mathbf{K}_{n}\right)(\varphi)$. It would seem to be irrational to change this value if $L$ is extended to a larger language by adding a set of propositional variables $\left\{b_{1}, \ldots, b_{k}\right\}$, all distinct from the variables of $L$, provided that we have not supplied any new knowledge. Following [10] we will formulate this as the following principle:

LI [Language Invariance Principle]. A social inference process $\mathcal{S}$ satisfies language invariance if whenever $L_{1}$ and $L_{2}$ are languages with $L_{1} \subseteq L_{2}$ and $\mathbf{K}_{1}, \ldots, \mathbf{K}_{n} \in C L_{1}$, then

$$
\mathcal{S}_{L_{1}}\left(\mathbf{K}_{1}, \ldots, \mathbf{K}_{n}\right)(\varphi)=\mathcal{S}_{L_{2}}\left(\mathbf{K}_{1}, \ldots, \mathbf{K}_{n}\right)(\varphi)
$$


for any $L_{1}$-sentence $\varphi$.

In the case when $n=1$ it is well known that several popular inference processes, including ME, satisfy LI (see [10] or p213 of [4] for details).

Following 10 we may also ask a different question in the same vein. What will happen if alongside the new propositional variables, new knowledge concerning these variables is also provided which contains no reference to the old variables. Again, it would seem to be rational that the value of a social inference process on a sentence that is formulated in the original language should not change. This leads us to

IIP [The Irrelevant Information Principle]. Let $L=L_{1} \cup L_{2}$ where $L_{1}$ and $L_{2}$ are disjoint propositional languages, and let $\mathbf{K}_{1}, \ldots, \mathbf{K}_{n}$ and $\mathbf{F}_{1}, \ldots, \mathbf{F}_{n}$ be knowledge bases formulated for the languages $L_{1}$ and $L_{2}$ respectively. Then for any $L_{1}$-sentence $\varphi$

$$
\mathcal{S}_{L}\left(\mathbf{K}_{1} \cup \mathbf{F}_{1}, \ldots, \mathbf{K}_{n} \cup \mathbf{F}_{n}\right)(\varphi)=\mathcal{S}_{L}\left(\mathbf{K}_{1}, \ldots, \mathbf{K}_{n}\right)(\varphi) .
$$

In the case when $n=1$ this principle plays a crucial role in the characterisation of ME in 12. Nevertheless, despite its intuitive plausibility this principle is in fact very hard to satisfy; indeed although ME satisfies it, almost all other commonly used (single agent) inference processes do not do so (see [10] and [4] for details).

IIP appears even harder for a social inference processes to satisfy. However, in this multi-agent case we might argue that this principle is just too strong. If knowledge provided by agents for the language $L_{2}$ is inconsistent then the addition of such new knowledge may provide us with more information on how strongly the agents disagree, which in turn may affect our evaluation of the knowledge concerning $L_{1}$. However, if the new knowledge does not change the level of disagreement as is the case when the new knowledge of all the agents is jointly consistent, then the principle of irrelevant information is arguably more justified. Accordingly we formulate:

CIIP [The Consistent Irrelevant Information Principle]. Let $L=L_{1} \cup L_{2}$ where $L_{1}$ and $L_{2}$ are disjoint propositional languages. Let $\mathbf{K}_{1}, \ldots, \mathbf{K}_{n}$ and $\mathbf{F}_{1}, \ldots, \mathbf{F}_{n}$ be knowledge bases formulated for the languages $L_{1}$ and $L_{2}$ respectively, and suppose that $\mathbf{F}_{1}, \ldots, \mathbf{F}_{n}$ are jointly consistent. Then for any $L_{1}$-sentence $\varphi$

$$
\mathcal{S}_{L}\left(\mathbf{K}_{1} \cup \mathbf{F}_{1}, \ldots, \mathbf{K}_{n} \cup \mathbf{F}_{n}\right)(\varphi)=\mathcal{S}_{L}\left(\mathbf{K}_{1}, \ldots, \mathbf{K}_{n}\right)(\varphi) .
$$

Assuming LI this last equation is equivalent to

$$
\mathcal{S}_{L}\left(\mathbf{K}_{1} \cup \mathbf{F}_{1}, \ldots, \mathbf{K}_{n} \cup \mathbf{F}_{n}\right)(\varphi)=\mathcal{S}_{L_{1}}\left(\mathbf{K}_{1}, \ldots, \mathbf{K}_{n}\right)(\varphi) .
$$

For instance, in the toy example of section 1 the information of both experts about a fault on the electric circuit is both consistent and a priori irrelevant to the probability that there is a fault on the valve. Hence if we want to know only the probability that there is a fault on the valve, then applying the CIIP we need consider only the fact that the first expert states that this probability is $40 \%$ and the second states that this probability is $80 \%$. 


\section{THE SOCIAL ENTROPY PROCESS}

In this section we define a particular social inference process formulated by the second author in [16] and 17]. The Social Entropy Process SEP, is defined by the following two stage process. At the first stage we define the set $\Delta_{L}\left(\mathbf{K}_{1}, \ldots, \mathbf{K}_{n}\right)$ as those probability functions $\mathbf{v}$ which globally minimise the sum of Kullback-Leibler divergences (crossentropies)

$$
\sum_{i=1}^{n} \mathrm{CE}\left(\mathbf{v}, \mathbf{w}^{(i)}\right)=\sum_{k=1}^{n} \sum_{j=1}^{J} v_{j} \log \frac{v_{j}}{w_{j}^{(k)}}
$$

subject only to the conditions that $\mathbf{w}^{(1)} \in V_{\mathbf{K}_{1}}, \ldots, \mathbf{w}^{(n)} \in V_{\mathbf{K}_{n}}$, where

$$
v_{j} \log \frac{v_{j}}{w_{j}^{(k)}}= \begin{cases}0 & \text { if } v_{j}=0 \text { and } w_{j}^{(k)}=0, \\ \infty & \text { if } v_{j} \neq 0 \text { and } w_{j}^{(k)}=0 .\end{cases}
$$

Recall that $v_{j}$ and $w_{j}^{(k)}$ stand for $\mathbf{v}\left(\alpha_{j}\right)$ and $\mathbf{w}^{(i)}\left(\alpha_{j}\right)$ respectively, where $\alpha_{j}$ is an atom and there are $J$ (logically inequivalent) atoms in $\operatorname{At}(L)$.

It is not difficult to see (see [17]) that $\Delta_{L}\left(\mathbf{K}_{1}, \ldots, \mathbf{K}_{n}\right)$ is nonempty if there is some atom $\alpha_{j}$ such that for no $i$ is it the case that for all $\mathbf{w} \in V_{\mathbf{K}_{i}} \mathbf{w}\left(\alpha_{j}\right)=0$. Under this condition $\Delta_{L}\left(\mathbf{K}_{1}, \ldots, \mathbf{K}_{n}\right)$ is well-defined. From now on we shall consider only $n$-tuples of knowledge bases $\mathbf{K}_{1}, \ldots, \mathbf{K}_{n}$ which satisfy this condition. Note that the definition of a social inference process is not much restricted by such an assumption.

In [17] it is proved that $\Delta_{L}\left(\mathbf{K}_{1}, \ldots, \mathbf{K}_{n}\right)$ is also a closed convex region of $\mathbb{D}^{L}$ and therefore there is a unique probability function in $\Delta_{L}\left(\mathbf{K}_{1}, \ldots, \mathbf{K}_{n}\right)$ having maximal entropy, and we will denote this function by $\mathbf{M E}_{L}\left(\Delta_{L}\left(\mathbf{K}_{1}, \ldots, \mathbf{K}_{n}\right)\right)$. Therefore, at the second stage of the definition we set $\mathbf{S E P}_{L}\left(\mathbf{K}_{1}, \ldots, \mathbf{K}_{n}\right)=\mathbf{M E}_{L}\left(\Delta_{L}\left(\mathbf{K}_{1}, \ldots, \mathbf{K}_{n}\right)\right)$. It is clear that $\mathbf{S E P}{ }_{L}$ coincides with $\mathbf{M E} \mathbf{E}_{L}$ in the case when $n=1$ and, it is straightforward to show that $\mathbf{S E P}$ is language invariant.

The set $\Delta_{L}\left(\mathbf{K}_{1}, \ldots, \mathbf{K}_{n}\right)$ is often a singleton and in that case the second stage is essentially redundant. For instance, this happens whenever $V_{\mathbf{K}_{k}}$ is a singleton for some $k$. The function which maps $\mathbf{K}_{1}, \ldots, \mathbf{K}_{n}$ to $\Delta_{L}\left(\mathbf{K}_{1}, \ldots, \mathbf{K}_{n}\right)$ is therefore called the weak social entropy process and is denoted by WSEP. WSEP may naturally be considered as a merging operator which merges the evidence of the $n$ agents into a single knowledge (or evidence) base without necessarily picking a unique social belief function ${ }^{1}$

For any $\mathbf{v} \in \Delta_{L}\left(\mathbf{K}_{1}, \ldots, \mathbf{K}_{n}\right)$ there is an $n$-tuple $\mathbf{w}^{(1)} \in V_{\mathbf{K}_{1}}, \ldots, \mathbf{w}^{(n)} \in V_{\mathbf{K}_{n}}$ minimizing $\sum_{k=1}^{n} \mathrm{CE}\left(\mathbf{v}, \mathbf{w}^{(k)}\right)$ defined in 11 . We will denote the set of all such $n$-tuples by $\Gamma_{L}\left(\mathbf{K}_{1}, \ldots, \mathbf{K}_{n}\right)$.

Lemma 4.1. The following are equivalent:

(i) The probability functions $\mathbf{v}, \mathbf{w}^{(1)}, \ldots, \mathbf{w}^{(n)}$ minimize (1) subject only to $\mathbf{w}^{(1)} \in$ $V_{\mathbf{K}_{1}}, \ldots, \mathbf{w}^{(n)} \in V_{\mathbf{K}_{n}}$.

(ii) $\mathbf{w}^{(1)}, \ldots, \mathbf{w}^{(n)}$ maximize $\sum_{j=1}^{J}\left(\prod_{k=1}^{n} w_{j}^{(k)}\right)^{\frac{1}{n}}$, subject only to $\mathbf{w}^{(1)} \in V_{\mathbf{K}_{1}}, \ldots$, $\mathbf{W}^{(n)} \in V_{\mathbf{K}_{n}}$, and $v_{j}=\frac{\left(\prod_{k=1}^{n} w_{j}^{(k)}\right)^{\frac{1}{n}}}{\sum_{j=1}^{J}\left(\prod_{k=1}^{n} w_{j}^{(k)}\right)^{\frac{1}{n}}}$ for all $j=1, \ldots, J$.

\footnotetext{
${ }^{1}$ This problematic is developed further in 1 .
} 
For a proof see [17]. We will define the maximal value of $\sum_{j=1}^{J}\left(\prod_{k=1}^{n} w_{j}^{(k)}\right)^{\frac{1}{n}}$ subject to $\mathbf{w}^{(1)} \in V_{\mathbf{K}_{1}}, \ldots, \mathbf{w}^{(n)} \in V_{\mathbf{K}_{n}}$ to be $\mathrm{M}_{L}\left(\mathbf{K}_{1}, \ldots, \mathbf{K}_{n}\right)$.

The lemma above implies that $\mathbf{S E P}_{L}$ coincides with the logarithmic (or "normalised geometric mean") pooling operator of decision theory (cf. [2]) in the very special case when each $V_{\mathbf{K}_{k}}$ defines a single probability function.

In addition to the above pleasing properties, SEP satisfies a set of natural principles listed in [16 and 17] similar to those shown to be satisfied by ME in 12. However these are almost certainly not sufficient to characterise SEP in the manner in which ME was characterised in 12 .

Furthermore, although SEP is language invariant, it does not satisfy the Irrelevant Information Principle IIP. A simple counterexample is provided by the following 2 .

Let $L_{1}=\left\{a_{1}\right\}, L_{2}=\left\{a_{2}\right\}$ and $L=L_{1} \cup L_{2}$. In the following we consider just two agents. Assume that the first agent possesses knowledge $\mathbf{K}_{1}=\left\{\mathbf{w}\left(a_{1}\right)=0.2\right\}, \mathbf{F}_{1}=$ $\left\{\mathbf{w}\left(a_{2}\right)=0.2\right\}$ and the second has knowledge $\mathbf{K}_{2}=\left\{\mathbf{w}\left(a_{1}\right)=0.3\right\}, \mathbf{F}_{2}=\left\{\mathbf{w}\left(a_{2}\right)=0.4\right\}$. Suppose that $\mathbf{w}^{(1)} \in V_{\mathbf{K}_{1} \cup \mathbf{F}_{1}}^{L_{1}}$ and $\mathbf{w}^{(2)} \in V_{\mathbf{K}_{2} \cup \mathbf{F}_{2}}^{L_{2}}$. We can identify $\mathrm{M}_{L}\left(\mathbf{K}_{1} \cup \mathbf{F}_{1}, \mathbf{K}_{2} \cup \mathbf{F}_{2}\right)$ defined above by maximizing the following expression for parameters $\mathbf{w}^{(1)}\left(a_{1} \wedge a_{2}\right)=x$ and $\mathbf{w}^{(2)}\left(a_{1} \wedge a_{2}\right)=y$ :

$$
\begin{aligned}
\mathrm{M}_{L}(x, y)=\quad & \sqrt{x y}+\sqrt{(0.2-x)(0.3-y)}+\sqrt{(0.2-x)(0.4-y)}+ \\
& +\sqrt{(0.6+x)(0.3+y)} .
\end{aligned}
$$

It is the matter of elementary analysis to prove that the above is strictly maximal for $x=0.12$ and $y=0.24$. Since $\mathrm{M}_{L}(0.12,0.24)=\sqrt{0.08}+\sqrt{0.48}$ it follows that

$$
\operatorname{SEP}_{L}\left(\mathbf{K}_{1} \cup \mathbf{F}_{1}, \mathbf{K}_{2} \cup \mathbf{F}_{2}\right)\left(a_{1}\right)=\frac{\sqrt{0.12 \cdot 0.24}+\sqrt{0.08 \cdot 0.06}}{\sqrt{0.08}+\sqrt{0.48}} .
$$

On the other hand

$$
\mathbf{S E P}_{L_{1}}\left(\mathbf{K}_{1}, \mathbf{K}_{2}\right)\left(a_{1}\right)=\frac{\sqrt{0.06}}{\sqrt{0.06}+\sqrt{0.56}} .
$$

Later, in corollary 5.4, we will show that SEP is language invariant; hence we also have that

$$
\operatorname{SEP}_{L}\left(\mathbf{K}_{1}, \mathbf{K}_{2}\right)\left(a_{1}\right)=\frac{\sqrt{0.06}}{\sqrt{0.06}+\sqrt{0.56}} .
$$

Since (2) and (3) are not equal it follows that SEP does not satisfy IIP.

Since IIP in its single agent form played a crucial role in the characterisation of ME, this failure could be interpreted as a significant criticism of SEP. However, while IIP may perhaps be too strong in the multi-agent case, we note that the weaker CIIP principle may still be regarded as a natural generalization of the single agent IIP since it also reduces to IIP for the case $n=1$.

\footnotetext{
${ }^{2}$ A counterexample to IIP for SEP was first found by Soroush Rafiee Rad (private communication, 2010).
} 
We shall say that the merging operator WSEP satisfies CIIP if, whenever $L=$ $L_{1} \cup L_{2}$ where $L_{1}$ and $L_{2}$ are disjoint propositional languages and $\mathbf{K}_{1}, \ldots, \mathbf{K}_{n}$ and $\mathbf{F}_{1}, \ldots, \mathbf{F}_{n}$ are knowledge bases formulated for the languages $L_{1}$ and $L_{2}$ respectively such that $\mathbf{F}_{1}, \ldots, \mathbf{F}_{n}$ are jointly consistent, then

$$
\left.\mathbf{W S E P}_{L}\left(\mathbf{K}_{1} \cup \mathbf{F}_{1}, \ldots, \mathbf{K}_{n} \cup \mathbf{F}_{n}\right)\right|_{L_{1}}=\mathbf{W S E P}_{L_{1}}\left(\mathbf{K}_{1}, \ldots, \mathbf{K}_{n}\right) .
$$

We prove that WSEP satisfies CIIP in the following section. However, except in the cases when $\Delta_{L_{1}}\left(\mathbf{K}_{1}, \ldots, \mathbf{K}_{n}\right)$ is a singleton, the question as to whether SEP also satisfies CIIP remains open.

We conclude this section by mentioning some other approaches to the problem of the integration of multi-agent probabilistic evidence which can be found in the literature. In the very special case when each agent specifies a single probability function, a procedure for combining this evidence into a single probability function is called a pooling operator by decision theorists. Pooling operators, and in particular the normalised geometric mean pooling operator to which SEP reduces in this special case, have been extensively studied from an axiomatic viewpoint (see e.g. [2]). However, except for the work previously cited $([16$, [17, [1] ), the more general problematic of a social inference process does not appear to have been investigated from an axiomatic standpoint. Nonetheless there exist a number of proposals for what are in essence particular social inference processes, sometimes defined in a somewhat different framework; in this context we should mention [6], [9], [13, [7], [8], and [15]. In particular in [8] and [15] results are proved about iterative convergence procedures based on Kullback-Leibler divergence, one of which yields particular points in $\Delta_{L}\left(\mathbf{K}_{1}, \ldots, \mathbf{K}_{n}\right)$. The connection between these algorithms and SEP merits further investigation.

\section{WSEP SATISFIES CIIP}

In what follows we will fix two distinct propositional languages $L_{1}=\left\{a_{1}, \ldots, a_{h_{1}}\right\}$ and $L_{2}=\left\{b_{1}, \ldots, b_{h_{2}}\right\}$. Let $L=L_{1} \cup L_{2}$ and let $\operatorname{At}\left(L_{1}\right)=\left\{\alpha_{1}, \ldots, \alpha_{J}\right\}$ and $\operatorname{At}\left(L_{2}\right)=$ $\left\{\beta_{1}, \ldots, \beta_{I}\right\}$.

For $\mathbf{r} \in S L$, to simplify the notation we will often denote $\left.\mathbf{r}\right|_{L_{1}}\left(\alpha_{j}\right)$ by $r_{j}$. We will also denote the conditional probability function $\mathbf{r}\left(\beta_{i} \mid \alpha_{j}\right)$ by $r_{i \mid j}$. It follows that $r_{j i}=r_{j} . r_{i \mid j}$, i.e. the value $r_{j i}$ can be computed as the product of the projection of $\mathbf{r}$ to $L_{1}$ on the $L_{1}$-atom $\alpha_{j}$ and the conditional probability $\mathbf{r}\left(\beta_{i} \mid \alpha_{j}\right)$.

Lemma 5.1. Let $w_{j}^{(k)} \geq 0$ be real numbers for all $1 \leq j \leq J$ and $1 \leq k \leq n$ where $k, j, J, n \in \mathbb{N}$. Then

$$
\sum_{j=1}^{J}\left(\prod_{k=1}^{n} w_{j}^{(k)}\right)^{\frac{1}{n}} \leq\left(\prod_{k=1}^{n} \sum_{j=1}^{J} w_{j}^{(k)}\right)^{\frac{1}{n}} .
$$

Equality holds if and only if either there are real constants $l^{(1)}>0, \ldots, l^{(n)}>0$ such that $l^{(1)}\left(w_{1}^{(1)}, \ldots, w_{J}^{(1)}\right)=l^{(2)}\left(w_{1}^{(2)}, \ldots, w_{J}^{(2)}\right)=\ldots=l^{(n)}\left(w_{1}^{(n)}, \ldots, w_{J}^{(n)}\right)$ or $\sum_{j=1}^{J} w_{j}^{(k)}=0$ for some $k$. 
This lemma is Hölder's inequality, see [3], and it will be very useful in the following proof.

Lemma 5.2. Let $\mathbf{K}_{1}, \ldots, \mathbf{K}_{n} \in C L_{1}, \mathbf{F}_{1}, \ldots, \mathbf{F}_{n} \in C L_{2}$ be such that $\mathbf{F}_{1}, \ldots, \mathbf{F}_{n}$ are jointly consistent.

(a) If $\mathbf{v} \in \Delta_{L_{1}}\left(\mathbf{K}_{1}, \ldots, \mathbf{K}_{n}\right)$ and $\mathbf{t}$ is an $L_{2}$-probability function such that $\mathbf{t} \in \bigcap_{i=1}^{n} V_{\mathbf{F}_{i}}$ then $\mathbf{v} \cdot \mathbf{t} \in \Delta_{L_{1} \cup L_{2}}\left(\mathbf{K}_{1} \cup \mathbf{F}_{1}, \ldots, \mathbf{K}_{n} \cup \mathbf{F}_{n}\right)$. In particular $\mathbf{F}_{1}, \ldots, \mathbf{F}_{n}$ could be empty in which case $\mathbf{t}$ can be arbitrary.

(b) Let $\mathbf{r} \in \Delta_{L_{1} \cup L_{2}}\left(\mathbf{K}_{1} \cup \mathbf{F}_{1}, \ldots, \mathbf{K}_{n} \cup \mathbf{F}_{n}\right)$. Then $\left.\mathbf{r}\right|_{L_{1}} \in \Delta_{L_{1}}\left(\mathbf{K}_{1}, \ldots, \mathbf{K}_{n}\right)$. Moreover $\mathrm{M}_{L_{1} \cup L_{2}}\left(\mathbf{K}_{1} \cup \mathbf{F}_{1}, \ldots, \mathbf{K}_{n} \cup \mathbf{F}_{n}\right)=\mathrm{M}_{L_{1}}\left(\mathbf{K}_{1}, \ldots, \mathbf{K}_{n}\right)$.

Proof. For a given $\mathbf{v} \in \Delta_{L_{1}}\left(\mathbf{K}_{1}, \ldots, \mathbf{K}_{n}\right)$ let $\left(\mathbf{p}^{(1)}, \ldots, \mathbf{p}^{(n)}\right) \in \Gamma_{L_{1}}\left(\mathbf{K}_{1}, \ldots, \mathbf{K}_{n}\right)$ be such that $v_{j}=\frac{\left(\prod_{k=1}^{n} p_{j}^{(k)}\right)^{\frac{1}{n}}}{\mathrm{M}_{L_{1}}\left(\mathbf{K}_{1}, \ldots, \mathbf{K}_{n}\right)}$. Then $\mathrm{M}_{L_{1}}\left(\mathbf{K}_{1}, \ldots, \mathbf{K}_{n}\right)=\sum_{j=1}^{J}\left(\prod_{k=1}^{n} p_{j}^{(k)}\right)^{\frac{1}{n}}$. For a given $\mathbf{r} \in \Delta_{L_{1} \cup L_{2}}\left(\mathbf{K}_{1} \cup \mathbf{F}_{1}, \ldots, \mathbf{K}_{n} \cup \mathbf{F}_{n}\right)$ let

$$
\left(\mathbf{w}^{(1)}, \ldots, \mathbf{w}^{(n)}\right) \in \Gamma_{L_{1} \cup L_{2}}\left(\mathbf{K}_{1} \cup \mathbf{F}_{1}, \ldots, \mathbf{K}_{n} \cup \mathbf{F}_{n}\right)
$$

be such that $r_{j i}=\frac{\left(\prod_{k=1}^{n} w_{j i}^{(k)}\right)^{\frac{1}{n}}}{\mathrm{M}_{L_{1} \cup L_{2}}\left(\mathbf{K}_{1} \cup \mathbf{F}_{1}, \ldots, \mathbf{K}_{n} \cup \mathbf{F}_{n}\right)}$.

Let us consider probability functions $\left.\mathbf{w}^{(1)}\right|_{L_{1}}, \ldots,\left.\mathbf{w}^{(n)}\right|_{L_{1}}$. We will denote $M=$ $\sum_{j=1}^{J}\left(\prod_{k=1}^{n} w_{j}^{(k)}\right)^{\frac{1}{n}}$. Then $M \leq \mathrm{M}_{L_{1}}\left(\mathbf{K}_{1}, \ldots, \mathbf{K}_{n}\right)$ since $\mathrm{M}_{L_{1}}\left(\mathbf{K}_{1}, \ldots, \mathbf{K}_{n}\right)$ is maximal. But by the lemma 5.1 also $\mathrm{M}_{L_{1} \cup L_{2}}\left(\mathbf{K}_{1} \cup \mathbf{F}_{1}, \ldots, \mathbf{K}_{n} \cup \mathbf{F}_{n}\right) \leq M$, hence

$$
\mathrm{M}_{L_{1} \cup L_{2}}\left(\mathbf{K}_{1} \cup \mathbf{F}_{1}, \ldots, \mathbf{K}_{n} \cup \mathbf{F}_{n}\right) \leq \mathrm{M}_{L_{1}}\left(\mathbf{K}_{1}, \ldots, \mathbf{K}_{n}\right) .
$$

(a) Let $\mathbf{t} \in \bigcap_{i} V_{\mathbf{F}_{i}}$. We are going to prove that

$$
\left(\mathbf{p}^{(1)} \cdot \mathbf{t}, \ldots, \mathbf{p}^{(n)} \cdot \mathbf{t}\right) \in \Gamma_{L_{1} \cup L_{2}}\left(\mathbf{K}_{1} \cup \mathbf{F}_{1}, \ldots, \mathbf{K}_{n} \cup \mathbf{F}_{n}\right) .
$$

It is easy to see that $\mathbf{p}^{(1)} \cdot \mathbf{t}, \ldots, \mathbf{p}^{(n)} \cdot \mathbf{t}$ satisfy $\mathbf{K}_{1} \cup \mathbf{F}_{1}, \ldots, \mathbf{K}_{n} \cup \mathbf{F}_{n}$ respectively. Moreover,

$$
\sum_{j=1, \ldots, J, i=1, \ldots, I}\left(\prod_{k=1}^{n} p_{j}^{(k)} t_{i}\right)^{\frac{1}{n}}=\sum_{j=1, \ldots, J, i=1, \ldots, I}\left(\prod_{k=1}^{n} p_{j}^{(k)}\right)^{\frac{1}{n}} t_{i}=\mathrm{M}_{L_{1}}\left(\mathbf{K}_{1}, \ldots, \mathbf{K}_{n}\right)
$$

since $\sum_{i=1}^{I} t_{i}=1$. But from 5 we already know that $\mathrm{M}_{L_{1} \cup L_{2}}\left(\mathbf{K}_{1} \cup \mathbf{F}_{1}, \ldots, \mathbf{K}_{n} \cup \mathbf{F}_{n}\right) \leq$ $\mathrm{M}_{L_{1}}\left(\mathbf{K}_{1}, \ldots, \mathbf{K}_{n}\right)$ hence (6) is proved.

(b) By (a) and (5) we have

$$
\mathrm{M}_{L_{1} \cup L_{2}}\left(\mathbf{K}_{1} \cup \mathbf{F}_{1}, \ldots, \mathbf{K}_{n} \cup \mathbf{F}_{n}\right)=M=\mathrm{M}_{L_{1}}\left(\mathbf{K}_{1}, \ldots, \mathbf{K}_{n}\right)
$$

hence

$$
\sum_{j=1, \ldots, J, i=1, \ldots, I}\left(\prod_{k=1}^{n} w_{j i}^{(k)}\right)^{\frac{1}{n}}=\sum_{j=1}^{J}\left(\prod_{k=1}^{n} \sum_{i=1}^{I} w_{j i}^{(k)}\right)^{\frac{1}{n}}
$$


By lemma 5.1 this equality could only occur if for each $j$ there are real constants $l_{j}^{(1)}>$ $0, \ldots, l_{j}^{(n)}>0$ such that the proportionality

$$
l_{j}^{(1)}\left(w_{j 1}^{(1)}, \ldots, w_{j I}^{(1)}\right)=l_{j}^{(2)}\left(w_{j 1}^{(2)}, \ldots, w_{j I}^{(2)}\right)=\ldots=l_{j}^{(n)}\left(w_{j 1}^{(n)}, \ldots, w_{j I}^{(n)}\right)
$$

holds, or $w_{j}^{(k)}=\sum_{i=1}^{I} w_{j i}^{(k)}=0$ holds for some $k$.

Let us consider the coefficient $j$ to be fixed. If $w_{j}^{(k)}=0$ for every $k$ let $\mathbf{q} \cdot \mid j$ be an arbitrary $L_{2}$-probability function with value on $i$-th atom denoted as $q_{i \mid j}$. Otherwise for $\bar{k}$ such that $w_{j}^{(\bar{k})} \neq 0$ let us define

$$
q_{i \mid j}=\frac{w_{j i}^{(\bar{k})}}{w_{j}^{(\bar{k})}}
$$

Obviously,

$$
\sum_{i=1}^{I} q_{i \mid j}=\sum_{i=1}^{I} \frac{w_{j i}^{(\bar{k})}}{\sum_{i=1}^{I} w_{j i}^{(\bar{k})}}=1
$$

and hence $\mathbf{q} \cdot \mid j$ is a well defined $L_{2}$-probability function. Notice that thanks to proportionality the definition does not depend on the choice of $\bar{k}$ :

$$
\frac{l_{j}^{(\bar{k})} w_{j i}^{(\bar{k})}}{l_{j}^{(\bar{k})} \sum_{i=1}^{I} w_{j i}^{(\bar{k})}}=\frac{l_{j}^{(k)} w_{j i}^{(k)}}{l_{j}^{(k)} \sum_{i=1}^{I} w_{j i}^{(k)}} .
$$

In other words

$$
w_{j i}^{(k)}=w_{j}^{(k)} q_{i \mid j} .
$$

By (7) the projections to $L_{1}$ satisfy

$$
\left(\left.\mathbf{w}^{(1)}\right|_{L_{1}}, \ldots,\left.\mathbf{w}^{(n)}\right|_{L_{1}}\right) \in \Gamma_{L_{1}}\left(\mathbf{K}_{1}, \ldots, \mathbf{K}_{n}\right) .
$$

Then for $L_{1}$-probability function $\mathbf{v}$ defined by $v_{j}=\frac{\left(\prod_{k=1}^{n} w_{j}^{(k)}\right)^{\frac{1}{n}}}{\sum_{j=1}^{J}\left(\prod_{k=1}^{n} w_{j}^{(k)}\right)^{\frac{1}{n}}}$ we have that $\mathbf{v} \in \Delta_{L_{1}}\left(\mathbf{K}_{1}, \ldots, \mathbf{K}_{n}\right)$.

Moreover,

$$
r_{j i}=\frac{\left(\prod_{k=1}^{n} w_{j i}^{(k)}\right)^{\frac{1}{n}}}{\sum_{j=1}^{J} \sum_{i=1}^{I}\left(\prod_{k=1}^{n} w_{j i}^{(k)}\right)^{\frac{1}{n}}}=\frac{\left(\prod_{k=1}^{n} w_{j .}^{(k)} q_{i \mid j}\right)^{\frac{1}{n}}}{\sum_{j=1}^{J} \sum_{i=1}^{I}\left(\prod_{k=1}^{n} w_{j}^{(k)} q_{i \mid j}\right)^{\frac{1}{n}}}=v_{j} q_{i \mid j},
$$

where $r_{j} .=\sum_{i} v_{j} q_{i \mid j}=v_{j}$ and $r_{i \mid j}=\frac{r_{j i}}{r_{j}}=\frac{v_{j} q_{i \mid j}}{r_{j}}=q_{i \mid j}$ which gives us the required result that $\left.\mathbf{r}\right|_{L_{1}} \in \Delta_{L_{1}}\left(\mathbf{K}_{1}, \ldots, \mathbf{K}_{n}\right)$.

Theorem 5.3. WSEP satisfies LI and CIIP.

This follows easily from lemma 5.2. Moreover this together with the fact that $\mathbf{M E}$ is language invariant (see [10]) yields the following: 
Corollary 5.4. SEP is language invariant.

Theorem 5.5. SEP satisfies the CIIP in the special case when there is only one probability function in $\Delta_{L_{1}}\left(\mathbf{K}_{1}, \ldots, \mathbf{K}_{n}\right)$, say $\Delta_{L_{1}}\left(\mathbf{K}_{1}, \ldots, \mathbf{K}_{n}\right)=\{\mathbf{w}\}$. Note that by theorem 3.8 in [17] this holds whenever at least one of the agents has a knowledge base which fixes a probability function for $L_{1}$.

Proof. By lemma 5.2 (b) clearly

$$
\left.\mathbf{S E P}_{L_{1} \cup L_{2}}\left(\mathbf{K}_{1} \cup \mathbf{F}_{1}, \ldots, \mathbf{K}_{n} \cup \mathbf{F}_{n}\right)\right|_{L_{1}}=\left.\mathbf{r}\right|_{L_{1}}=\mathbf{w}=\mathbf{S E P}_{L_{1}}\left(\mathbf{K}_{1}, \ldots, \mathbf{K}_{n}\right) .
$$

\section{CONCLUSION}

In this paper we have sought to investigate the Irrelevant Information Principle in the context of multi-agent uncertain reasoning. While this principle plays a crucial role in an axiomatic characterization of $\mathbf{M E}$ given in [12, we have argued that the most obvious generalization of the Irrelevant Information Principle to the multi-agent context may be too strong. We have proposed an alternative generalization for a social inference process called the Consistent Irrelevant Information Principle (CIIP). We have described the promising social inference process SEP first formulated in [16] and its weaker counterpart, the merging operator WSEP. We have shown that WSEP satisfies CIIP and that SEP satisfies CIIP in many cases. The question as to whether SEP satisfies CIIP in general remains open.

\section{ACKNOWLEDGEMENT}

The authors are very grateful to Alena Vencovská for spotting a mistake in the original proof of lemma 5.2 Thanks are also due to the anonymous referees for their helpful comments.

The research leading to these results has received funding from the European Commission's Seventh Framework Programme [FP7/2007-2013] under grant agreement no 238381.

(Received ????)

\section{REFERENCES}

[1] M. Adamčík and G. M. Wilmers: Probabilistic Merging Operators. Logique et Analyse (2013), to appear.

[2] S. French: Group Consensus Probability Distributions: A Critical Survey. In: J. M. Bernardo, M. H. De Groot, D. V. Lindley, and A. F. M. Smith (Eds.), Bayesian Statistics, Elsevier, North Holland (1985), pp. 183-201.

[3] G. H. Hardy, J. E. Littlewood and G. Pólya: Inequalities. Cambridge University Press, 1934.

[4] P. Hawes: An Investigation of Properties of Some Inference Processes. PhD Thesis, The University of Manchester, Manchester 2007.

[5] E. T. Jaynes: Where do we Stand on Maximum Entropy? In: The Maximum Entropy Formalism, R. D. Levine and M. Tribus (eds.), M.I.T. Press, Cambridge (1979), MA. 
[6] G. Kern-Isberner and W. Rödder: Belief Revision and Information Fusion on Optimum Entropy. International Journal of Intelligent Systems 19 (2004), pp 837-857.

[7] J. Kracík: Cooperation Methods in Bayesian Decision Making with Multiple Participants. Ph.D. Thesis, Czech Technical University, Prague 2009.

[8] F. Matúš: On iterated averages of $I$-projections. Universität Bielefeld, Germany 2007.

[9] D. Osherson and M. Vardi: Aggregating disparate estimates of chance. Games and Economic Behavior 56 issue 1 (2006), pp. 148-173.

[10] J. B. Paris: The uncertain reasoner's companion. Cambridge University Press, Cambridge 1994

[11] J. B. Paris and A. Vencovská: On the Applicability of Maximum Entropy to Inexact Reasoning. International Journal of Approximate Reasoning 3 (1989).

[12] J. B. Paris and A. Vencovská: A Note on the Inevitability of Maximum Entropy. International Journal of Approximate Reasoning 4 (1990), pp. 183-224.

[13] J. B. Predd, D. N. Osherson, S. R. Kulkarni and H. V. Poor: Aggregating Probabilistic Forecasts from Incoherent and Abstaining Experts. Decision Analysis 5 No. 4 (2008), pp. 177-189.

[14] J. E. Shore and R. W. Johnson: Axiomatic Derivation of the Principle of Maximum Entropy and the Principle of Minimum Cross-Entropy. IEEE Transactions on Information Theory IT-26(1) (1980), pp. 26-37.

[15] J. Vomlel: Methods of Probabilistic Knowledge Integration. PhD thesis, Czech Technical University, Prague 1999.

[16] G. M. Wilmers: The Social Entropy Process: Axiomatising the Aggregation of Probabilistic Beliefs. In: Probability, Uncertainty and Rationality, edited by Hykel Hosni and Franco Montagna, 10 CRM series, Scuola Normale Superiore, Pisa (2010), pp. 87-104.

[17] G. M. Wilmers: Generalising the Maximum Entropy Inference Process to the Aggregation of Probabilistic Beliefs. available from http://manchester.academia.edu/GeorgeWilmers/Papers

Martin Adamčik, Room 2.226, Alan Turing Building, The University of Manchester, Oxford Road, M13 9PL Manchester, UK

e-mail:martin.adamcik@manchester.ac.uk

George Wilmers, Alan Turing Building, The University of Manchester, Oxford Road, M13 9PL Manchester, UK e-mail: george.wilmers@gmail.com 